\title{
PROGRAMA DE RISCO DE RADÔNIO PARA O BRASIL
}

\section{Souza Filho, O.A., CPRM/SGB, Rocha, Z., CDTN/CNEN} Copyright 2018, SBGf - Sociedade Brasileira de Geofísica

Este texto foi preparado para a apresentação no VIII Simpósio Brasileiro de Geofísica, Salinópolis, 18 a 20 de setembro de 2018. Seu conteúdo foi revisado pelo Comitê Técnico do VIII SimBGf, mas não necessariamente representa a opinião da SBGf ou de seus associados. É proibida a reprodução total ou parcial deste material para propósitos comerciais sem prévia autorização da SBGf.

\section{Abstract}

The Brazilian Radon Risk Map and the Radon Risk Program have the goal to subsidize government and stake holders to create directives to minimize the risk of exposure to radon. Radon and its progenies are the source of most of ionizing radiation throughout human life and after tobacco smoking, it is the second cause of lung cancer death according to World Health Organization. In Brazil, 22,424 deaths by lung cancer occurred in 2011 and it is the second cause of death in people over 50 y.o. age. The CPRM-SGB and CNEN-National Commission on Nuclear Energy will call for partners from geosciences and medical research centers and universities, local and federal health and environmental agencies and civil associations. Budget will be sought in national and international financial agencies. The four-phase program will last about 10 years. During the first two years the core methodology will be developed towards a radon-risk map based on radon measurements in dwellings (including lung-cancer patient's residences) and airborne and ground gamma-ray spectrometry surveys. The studyareas will start from where radon risk assessment already took place by initiatives from the CNEN, Fundação Osvaldo Cruz (FIOCRUZ) and universities. A long-term action plan will account for mitigation, continue indoor measurements and risk-assessment in building materials and budgets for continuous high-level research.

\section{Introdução}

O Radônio - Rn é um elemento natural radioativo, pertencente à família dos gases nobres. Origina-se do decaimento do urânio e tório, portanto, presente em suas séries naturais. $\mathrm{O}$ isótopo ${ }^{222} \mathrm{Rn}$ é o mais abundante, de maior duração, com meia-vida de 3,8 dias e é inerte. Os outros isótopos ${ }^{220} \mathrm{Rn}$ (Torônio) e ${ }^{219} \mathrm{Rn}$ (Actinônio) possuem meia-vida de segundos. Seus elementos-filhos, Polônio, Bismuto e Chumbo são sólidos e também inertes, mas instáveis, que continuam emitindo radiações Alfa, Beta e Gama.

O Radônio é encontrado em rochas, solos e materiais de construção que contenham Urânio, Tório e Rádio. Elevadas concentrações podem ocorrer em variedades de rochas ígneas graníticas e alcalinas, arenitos e areias monazíticas, folhelhos, calcários, depósitos de urânio, carvão, minerais metálicos e outros. Valores equivalentes podem estar nos solos derivados destas rochas.

O Radônio e Torônio respondem por $55 \%$ da radiação que o ser humano recebe ao longo de sua vida. (ICRP,
2009). Eles se dispersam na atmosfera, mas acumulamse em ambientes fechados, dentro de residências e prédios com fundação direta sobre o solo. Materiais de construção civil e ornamentação (pisos e paredes em granito) podem acrescentar doses extras de radiação. $E$ não o percebemos por que o gás e seus subprodutos são incolores, insípidos e inodoros.

O radônio e seus elementos-filhos são reconhecidos pela Organização Mundial de Saúde (WHO, 2009) e agências nacionais de saúde como a segundo maior causador de câncer de pulmão, atrás do tabagismo. Junto com elementos cancerígenos do tabagismo, eles liberam partículas radioativas que, ao serem inaladas, acumulamse nas vias aéreas e podem provocar a multiplicação descontrolada ou morte das células. Ao ser ingerido com água, anteveem-se complicações de câncer de estômago, embora com menor risco. Mesmo em não fumantes existe o risco de desenvolvimento da doença (Ruano-Ravina et al., 2017).

Um comparativo de 1990 a 2015 mostra que no Brasil, o câncer de pulmão é a segunda causa de óbito por neoplasias em pessoas acima de 50 anos de idade. A mortalidade é maior nos homens e nos estados RS, SC, AM e AC e, aumentou nos estados do CE, RN e BA (Guerra et al, 2017). O INCA (2018) registrou 22.424 mortes em 2011. A sobrevida de doentes é inferior a 10 $\%$, tornando-se a principal causa de mortes evitáveis no século XX, com aumento do risco para hereditariedade da doença ou desenvolvimentos de outros tipos de câncer (INCA, 2017).

Nos países desenvolvidos, há normativas sobre mapeamento de risco, prevenção, mitigação do risco e ventilação durante e após a construção de habitações e campanhas de esclarecimento da população (Ex.: WHO, 2009, EURATOM, 2013). Os planos de ação são elaborados por comitês nacionais e estaduais, envolvendo poderes executivo e legislativo, agências geológicas, ambientais e de saúde e organizações médicas e civis. Os mapas de risco de exposição são gerados a partir de medições in loco de radônio e medições gamaespectrométricas. Como exemplo de impacto social, a estratégia americana (EPA-US, 2015) contempla até 2020 a redução à exposição ao radônio em 5 milhões de lares, evitando de cerca de 3.220 mortes anuais.

No Brasil, as pesquisas acadêmicas e ocupacionais relacionam o índice de câncer com radiações naturais do subsolo (causa geogênica) e artificiais, (Veiga, 2004; Godoy et al., 2011; Campos, et al., 2013; INCA, 2013; Merola, 2017). O Instituto de Radioproteção e DosimetriaIRD/CNEN é responsável pela metrologia legal e a DRS/CNEN pela inspeção regulatória das radiações ionizantes. O IRD/CNEN executa um projeto de medição de radônio em minas subterrâneas (Gouvea et al., 2014). Todavia, a nível nacional, não há mapas indicativos de 
áreas de risco, campanhas de esclarecimento e restritos são os estudos em materiais utilizados na construção civil (CDTN e IPEN/CNEN, UTFPR e USP).

O Programa Brasileiro de Risco de Exposição ao Radônio terá por objetivo principal suprir a falta de indicadores da exposição natural ao radônio e de prover o estado com subsídios para iniciar planos de ação e otimização das políticas de câncer.

\section{Metodologia}

O programa deverá ser executado em conjunto com a CNEN, num prazo máximo de conclusão de 10 anos. Também serão chamados para participar o Ministério da Saúde, de Minas e Energia, do Meio Ambiente, da Ciência e Tecnologia, as associações oncológicas, universidades, secretarias de saúde. Entendimentos com as agências estaduais, CNPq, FINEP e os ministérios anteriormente citados ocorrerão para criarem dotações orçamentárias exclusivas e linhas diretas de financiamento para garantir a continuidade em longo prazo do mapeamento, aparelhamento de laboratórios, manutenção de equipamentos e bolsas de estudos e pesquisas de ponta pelas instituições em geociências e em saúde.

O orçamento estimado de custeio para a CPRM nos dois primeiros anos, excluindo custo de pessoal, é de $R \$ 200$ mil, que contempla etapas de campo e viagens, análises laboratoriais e equipamentos. Além da utilização compartilhada de equipamentos dos centros brasileiros e estrangeiros de pesquisas, estão previstas a compra de detetores de traços nucleares de estado sólido (SSNTD), CR-39 para medidas de longo prazo. No IRD/CNEN, há a possibilidade de se construir protótipos e aferi-los, reduzindo o custo para as centenas de unidades necessárias na primeira fase. Para medições de curto prazo, espera-se investir em detetores de eletretos, como no modelo RADELEC Inc.

A disseminação do conhecimento, avaliação da metodologia de representação do mapa de risco e a colaboração internacional serão estimulados por meio de simpósios anuais e encontros regionais entre a comunidade científica e gestores públicos e sociedade.

Fase 1 - A primeira fase durará 2 anos, quando serão definidas as entidades financiadoras e as executoras, detalhamento da metodologia e a adequação de medidores de radônio. O mapeamento das áreas de risco envolverá medições de radônio e gamaespectrométricas (concentrações de Potássio-K, Urânio-eU, de Tório-eTh) nas áreas habitacionais dos pacientes identificados no Registro de Câncer de Base Populacional. A informação in situ será regionalizada a partir da correlação com dados geofísicos radiométricos e geoquímicos, baseados em formulações e relações estatísticas padronizadas internacionalmente. Inicialmente serão apresentados mapas de risco para o Leste do Paraná e áreas com estudos prévios de radioatividade e geoquímica ambiental realizados pela CNEN, Fundação Osvaldo Cruz (FIOCRUZ) e universidades em Belo Horizonte, São Paulo, Salvador, Lages Pintada/Rn, Poços de Caldas e em depósitos minerais.
Fase 2 - Expansão do mapeamento para outros estados mediante acordos de cooperação, priorizando áreas mais populosas. A metodologia será aferida e caso necessário, haverá adaptação conforme as características regionais.

Fase 3 - Com base nas informações geradas nas fases 1 e 2, serão constituídos os comitês nacional e estaduais para elaborar um plano de ação e normativas quanto a prevenção e mitigação de exposição ao radônio e outros elementos associados.

Fase 4 - Progressivo recobrimento do país com mapas de risco e expansão dos comitês estaduais.

O método geofísico radiométrico estuda as concentrações dos radioelementos ${ }^{40} \mathrm{~K},{ }^{238} \mathrm{U}$ e ${ }^{232} \mathrm{Th}$ e a contagem total de radiação Gama (CT) emitidas por material, solo ou rocha. Os gamaespectrômetros medem as concentrações destes elementos a partir da quantidade incidente de radiação dentro dos intervalos de energia específicas de cada elemento. Portanto os dados aerogeofísicos serão de suma importância para a geração de mapas considerando a abrangência do território brasileiro.

Ênfase será dada as regiões mais populosas ou de intensa atividade agroindustrial. A gamaespectrometria aeroportada de alta resolução e a geoquímica de rocha, solo e agua possibilitarão o mapeamento de cerca da metade do território (Figura 1), mormente as regiões Sudeste e Nordeste, Centro-Oeste e as principais províncias minerais.

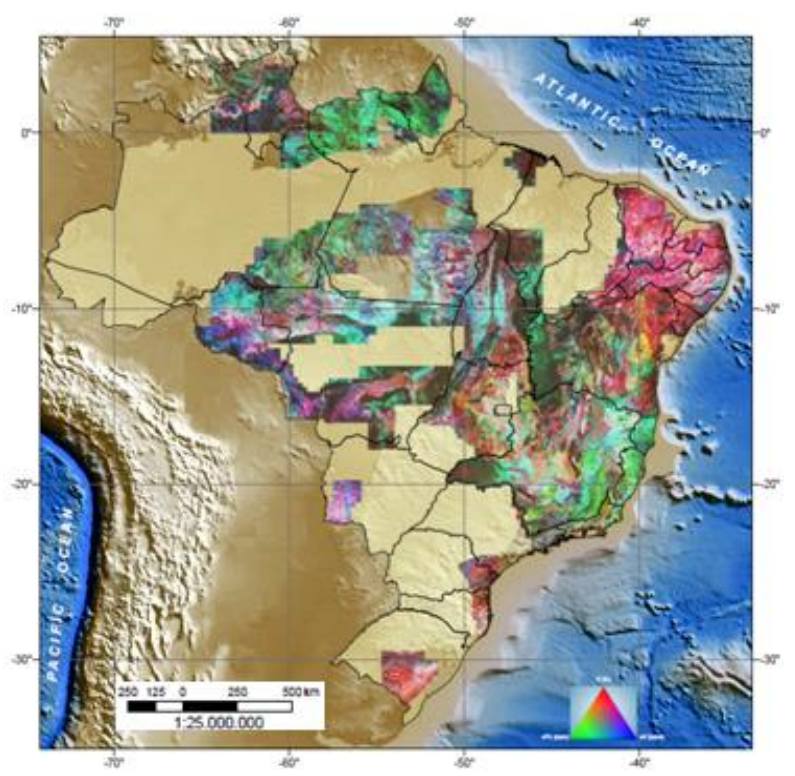

Figura 1 - Áreas possíveis de mapeamento de risco de radônio baseadas na cobertura aerogeofísica gamaespectrométrica disponível para o Brasil (Modif. de Correa, 2016).

Serão adotadas as recomendações da IAEA (2018) para levantamentos de radônio in situ e adaptados os procedimentos tomados nos diversos países. As formulações internacionalmente aceitas, que relacionam 
as concentrações de $\mathrm{K}$, eU, eTh e contagem total com as taxas de doses e de risco à radiação são:

a) Taxa de Dose Total (DR, nGy h${ }^{-1}$; IAEA, 2010):

$\mathrm{DR}=13,078^{\star} \mathrm{K}+5,675^{\star} \mathrm{eU}+2,494^{\star} \mathrm{eTh}$; sendo $\mathrm{K}(\%)$, eU (ppm); eTh (ppm)

b) Dosagem Efetiva Anual de exposição (DAR, $\mu \mathrm{Sv} \mathrm{y}^{-1}$ ) ao K, eU, eTh a $1 \mathrm{~m}$ do solo (Ray and Stick, 2015):

$\mathrm{DAR}=320+52,6^{*}\left[\left(\mathrm{~K}^{*} 1,505\right)+\left(\mathrm{eU} \mathrm{U}^{\star} 0,625\right)+\left(\mathrm{eTh}^{\star} 0,310\right)\right]$, sendo: constantes 320 é a soma da radiação cósmica e radiação interna de um corpo e a constante 52,6 a conversão de medidas gamaespectrométricas de: $\mu \mathrm{R}$ $\mathrm{y}^{-1}=>(x 0,6)$ mrem $\mathrm{y}^{-1}=>S v \mathrm{y}^{-1}\left(\mathrm{~Sv} \mathrm{y}^{-1}=100 \mathrm{mrem}\right)$. (Grastly et al., 1984; Pascholati et al., 1997).

c) Dose Efetiva Anual, de radônio medido em ambiente interno (USNCEAR, 2000; Quarto et al., 2015):

$\mathrm{H}\left(\mathrm{mSv} \mathrm{a}^{-1}\right)=\mathrm{C} \times \mathrm{F} \times \mathrm{O} \times \mathrm{T} \times \mathrm{D}$, onde $\mathrm{C}=$ concentração média de radônio; $F$ fator de equilíbrio em ambientes fechados ( $\mathrm{F} \sim 0,4) ; \mathrm{O}=$ fator de ocupação $(\mathrm{O} \sim 0,8) ; \mathrm{T}=$ horas em um ano $(\mathrm{T}=8.760$ $\left.h \mathrm{a}^{-1}\right) ; \mathrm{D}=$ fator de conversão de dose $\left(\mathrm{F} \sim 14^{*} 10^{-6}\right.$

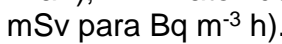

d) Atividade equivalente de Rádio ( $\mathrm{Ra}_{\mathrm{Eq}}$ em $\mathrm{Bq} \mathrm{kg}^{-1}$ ) um indicador ponderado da soma das atividades de $U^{238}$, $\mathrm{Th}^{232}$ e $\mathrm{K}^{40}$, avalia o dano radiológico por exposição a materiais de construção.

$\mathrm{RaEq}_{\mathrm{E}}=\mathrm{Cu}_{\mathrm{u}}+143 \mathrm{C}_{\mathrm{Th}} 0,077 \mathrm{C}_{\mathrm{k}}$; que é equivalente a a taxa de dose de $10 \mathrm{~Bq} \mathrm{~kg}^{-1}{ }^{226} \mathrm{Ra}+7 \mathrm{~Bq} \mathrm{~kg}^{-1}{ }^{232} \mathrm{Th}+$ $130 \mathrm{~Bq} \mathrm{~kg}^{-1}{ }^{40} \mathrm{~K}$

$\mathrm{O}$ valor de $370 \mathrm{~Bq} \mathrm{~kg}{ }^{-1}$ de atividade equivalente de Rádio ( 1,5 $\left.\mathrm{mSv} \mathrm{a}^{-1}\right)$ é a dose máxima de segurança na construção civil (Beretka e Matthew, 1985).

e) Índice de risco externo (UNSCEAR, 2000), mede a exposição em ambiente aberto a radiações gama a longo prazo. A ECRP (1999) recomenda $\mathrm{H}_{\mathrm{ex}}<1$ :

$\mathrm{H}_{\mathrm{ex}}=\mathrm{C}_{\mathrm{Ra}} / 370+\mathrm{C}_{\mathrm{Th}} / 259+\mathrm{C}_{K} / 4810$

f) Dose Média Ponderada pela População (UNSCEAR, 2000): $D p=\left(\mathrm{C}_{p} \times T \times f \times k\right) / 10^{6}$,

onde $D p$ (mSv/ano); $C p=$ concentração ponderada pela população em $\mathrm{Bq} / \mathrm{m}^{3}, T=$ número de horas por ano com um fator de ocupação do $80 \%, f=$ fator de equilíbrio $=0,4, k=$ constante de proporcionalidade para conversão $\mathrm{nSv} / \mathrm{h} / \mathrm{Bq} / \mathrm{m}^{3}$

g) Cálculo de Casos de Câncer Associados ao Radônio, baseada em formulações como em Darby et al. (2005), Elío et al. (2018).

Medições de radônio in situ no ar deverão ser realizadas com medidores passivos de traços nucleares do tipo Policarbonato Lexan e CR-39 para medidas de longo prazo e situados em um ou dois ambientes da residência. Medidas no ar de curto prazo serão realizadas com câmaras de ionização de eletretos (SST), seguindo protocolos da EPA (2009).
Locais de prolongada permanência (ocupacional e moradia) serão calculado o Fator de Equilíbrio no ar: razão entre a concentração equivalente de equilíbrio (CEE, medido com o DOSEman) e a concentração de radônio ( $\mathrm{C}_{\mathrm{Rn}}$, medida com o AlfaGUARD). O equipamento RAD7 medirá o radônio na água de poços, para avaliar o risco proveniente de aquíferos.

O Plano de Ação conterá instruções para programas a nível regional e específico em ambientes ocupacionais e áreas de risco de exposição, incorporadas em:

- recomendações de monitoramento, expansão dos testes in situ e remediação com foco nas residências, escolas e hospitais e ambientes ocupacionais de risco;

- cooperação com o setor de construção civil com incentivo ao uso de materiais de baixa taxa de exalação de radônio e disseminação das técnicas de mitigação e valoração e certificação das empresas que usam tais materiais e de profissionais que avaliarão;

\section{Resultados Esperados}

Mostraremos exemplos de mapas de risco e a metodologia base de alguns países.

Nos Estados Unidos, o programa de risco de radônio foi iniciado após a Public Law 100-551 de 1988. O mapa final de referência à população e ao gestor foi desenvolvido pela Agência de Proteção Ambiental-EPA e - Serviço Geológico-USGS a partir dados coletados e estudos prévios na escala de condados e estados federativos. O mapa designa três Zonas de Risco e cada zona reflete a média de radônio medido a curto prazo provável de ocorrer em habitações (residências ou prédios) sem a implementação de métodos de controle de radônio. (Figura 2; EPA, 2018, USGS, 1993).

Zona 1, de maior potencial (> $4 \mathrm{pCi} / \mathrm{L}$ ); Zona 2, de moderado potencial (2 a $4 \mathrm{pCi} / \mathrm{L}$ ); Zona 3 , de baixo potencial $(<2 \mathrm{pCi} / \mathrm{L})$.

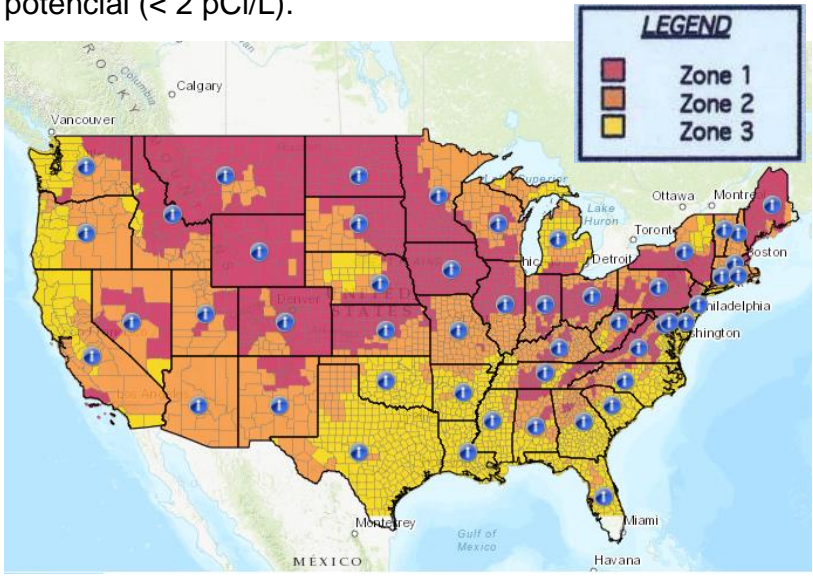

Figura 2 - Zonas de risco de radônio para os Estados Unidos. (Modificado. EPA, 2018).

O mapa americano foi concebido a partir de: medições de radônio em ambientes fechados (até 7 dias de duração); geologia; gamaespetrometria aeroportada; 
permeabilidade de solo; tipo de fundação. De acordo com o EPA, o mapa auxilia na definição de áreas prioritárias de alocação de recursos e implementar medidas de contenção de radônio nas construções. Entretanto, nenhuma das três zonas determina se um lar deveria ser testado para radônio, pois apenas a medição in situ define se a residência está livre dessa ameaça.

Após estudos prévios, a Irlanda realizou entre 1992 e 1999 medições de radônio (duração de 12 meses) em 11.319 residências, distribuídas numa malha geográfica de $10 \mathrm{~km}^{2}$. Os níveis de radônio variaram entre $10 \mathrm{e}$ $1,924 \mathrm{~Bq} / \mathrm{m}^{3}$. O nível de referência foi estabelecido em $200 \mathrm{~Bq} / \mathrm{m}^{3}$ e a célula da malha em que $10 \%$ das residências excedesse 0 valor de $200 \mathrm{~Bq} / \mathrm{m}^{3}$ era considerada de alto risco. Comparando-se o número de residências em cada célula, estimou-se que 91.019 casas (7\% do total) tinham concentrações de superiores à referência e que a concentração média ponderada pela população era de $91 \mathrm{~Bq} / \mathrm{m}^{3}$. (RPII, 2002, Figura 3).

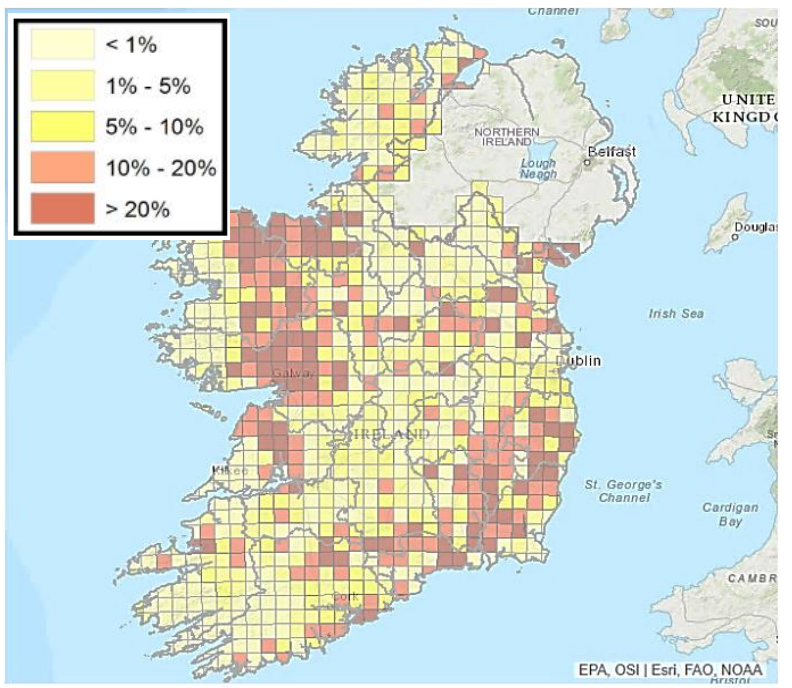

Figura 3 - Risco de radônio para a Irlanda. Valores em porcentagem de residências com valor superior à referência de 200 Bq/m³. (Modif. EPA-IE, 2018).

A Irlanda do Norte lançou equivalente produto em 2015, utilizando séries históricas e medições em 23.000 lares e posterior balizamento com limites geológicos numa malha com células de $1 \mathrm{~km}$. Nas residências, as medições foram realizadas com dois detectores passivos de integração (na sala e num quarto) por 3 meses e normalizados para temperatura/época do ano. O mapa define áreas com probabilidade de exceder em $1 \%$ ou superior, o limite de ação de $200 \mathrm{~Bq} / \mathrm{m}^{3}$, nas quais as habitações devem realizar medições in situ e medidas protetivas e de isolamento em novas construções. (Figura 4; Daraktchieva et al., 2015).

A Noruega possui uma das maiores concentrações de radônio do mundo. Em até $9 \%$ de todas as 175.000 residências, os valores estão acima do limite de 100 $\mathrm{Bq} / \mathrm{m}^{3}$. O país iniciou levantamentos residenciais de radônio nos anos 80 s. O mapa de risco desenvolvido pelo Serviço Geológico e Autoridade de Proteção à
Radiação baseia-se em medições de radônio no ar (6.326 residências), dados aerogamaespectrométricos, mapas geológico, de solos e depósitos inconsolidados. (Figuras 4 e 5; NGU, 2017; Watson et al., 2104).

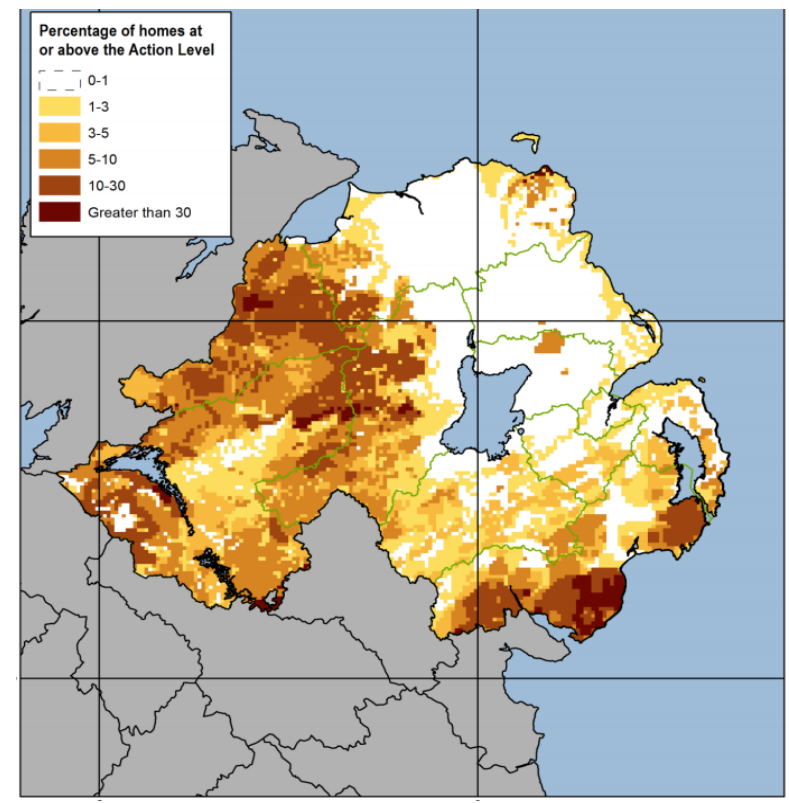

Figura 3 - Risco de radônio na Irlanda do Norte (Daraktchieva et al., 2015).

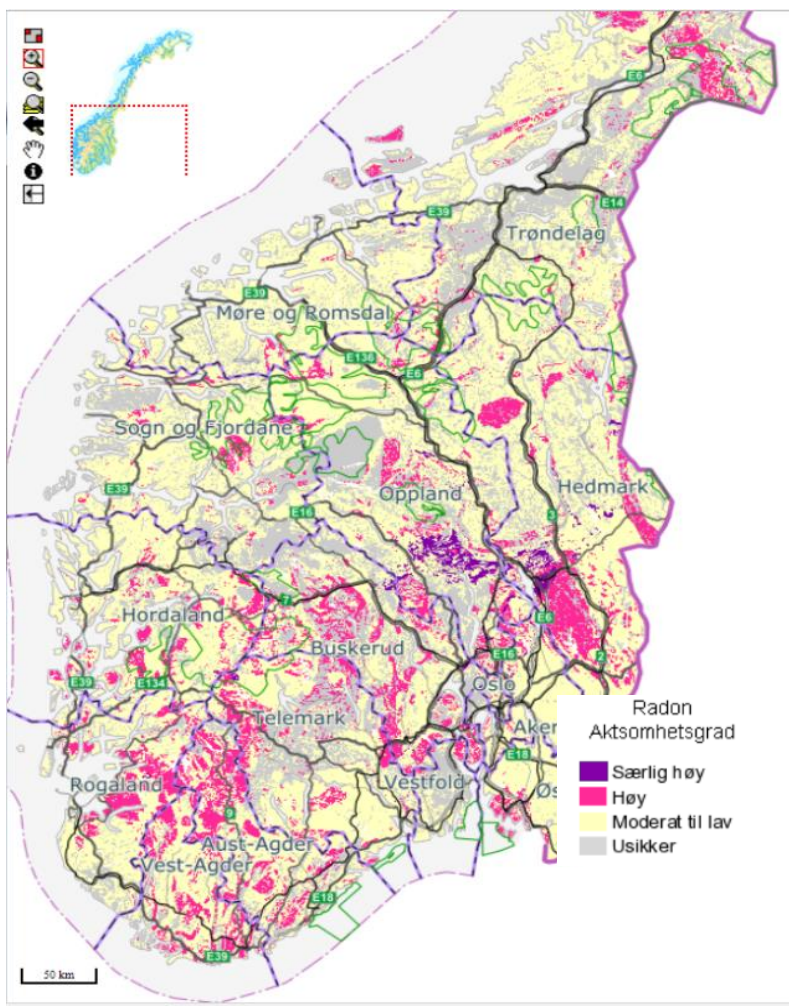

Figura 4 - Risco de radônio na Noruega. (Modif. NGU, 2017). Risco: muito alto (roxo); alto (rosa); moderado a baixo (amarelo); incerto (cinza). 


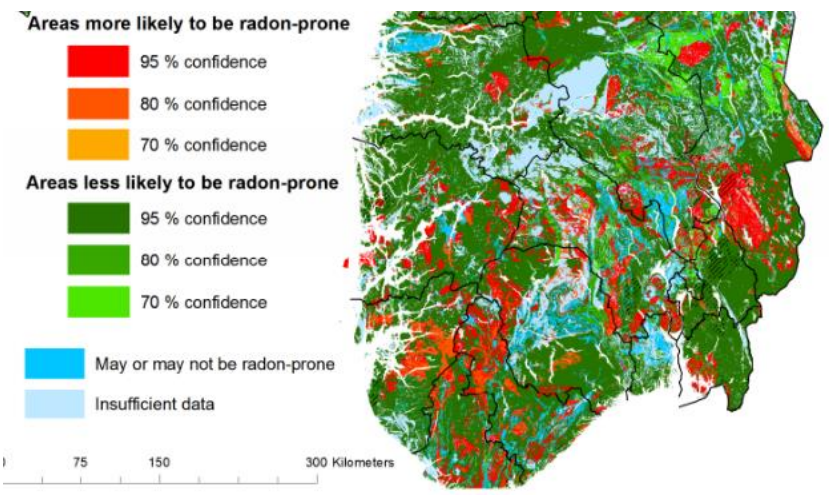

Figura 5 - Versão de 2014 para o mapa de risco da Noruega. (Modif. Watson et al., 2014).

\section{Discussão e Conclusões}

O risco de desenvolvimento de câncer de pulmão associado à exposição de radônio no ar que se respira, é reconhecidamente elevado, não importando entre fumantes e não fumantes. Mesmo sendo considerada doença evitável, está dentre as mais letais, com uma sobrevida inferior a $10 \%$ após cinco anos de manifestação nos países em desenvolvimento. Em 2015 no Brasil, ocorreram 26.492 óbitos e, estimam-se 31.270 novos casos de câncer para 2018-2019 somando-se homens e mulheres. Isto reflete-se num "custo saúde" de $\mathrm{R} \$ 39,4$ bi em despesas médicas e $\mathrm{R} \$ 17,5$ bi em custos indiretos (perda de produtividade, causada pela incapacitação ou morte prematura). (INCA, 2018)

Não há no Brasil uma estimativa de casos diretamente relacionados ao radônio, embora haja menções de risco em relatórios do INCA (2018), e em estudos acadêmicos. À título de comparação, na Noruega, corresponde entre 5 - 15\% (100 - a 300 casos) do total registrado anualmente; Jensen et al., 2004) e os Estados Unidos calculam 21.000 mortes por ano (EPA-US, 2015).

O Programa de Risco de Exposição ao Radônio no Brasil está ancorado em três produtos principais: mapa nacional de risco de radônio; plano de ação e normas e regulamentações para prevenção, mitigação e uso de materiais na construção civil e riscos ocupacionais. Almeja estabelecer pilares e políticas de longo prazo para eliminar casos evitáveis de câncer de pulmão induzido por radônio. E desta maneira, reduzir parte dos milhares de dezenas de novos casos de câncer a cada ano no Brasil.

\section{Agradecimentos}

Os autores agradecem às respectivas instituições por apoiarem essa iniciativa que pode ter grande impacto à saúde pública.

\section{Referências}

Beretka, J., Matthew, P. J., 1985. Natural radioactivity of Australian building materials, industrial wastes and byproducts. Health Physcis, 48: 87-95.

Campos et al. (05 Coautores), 2013. O gás radônio doméstico e a radioatividade natural em terrenos metamórficos: o caso do município de Lucrecia (Rio Grande do Norte, Brasil). Rev. Geol., v. 26, n. 2:85-93.

Correa, R. T., 2016. Radiometric Map of Brazil (first edition). Scale 1: 25,000,000. Brasília, CPRM Geological Survey of Brazil.

Darby et al. (25 coautores), 2005. Radon in homes and risk of lung cancer: collaborative analysis of individual data from 13 European case-control studies. BMJ, 330: p. $223,10.1136 / \mathrm{bmj} .38308 .477650 .63$.

Daraktchieva et al. (08 coautores), 2015. Radon in Northern Ireland: Indicative Atlas. Cooperação Public Health England, British Geol Survey, Northern Ireland Environ. Agency. PHE-CRCE-017. Disponível em https://assets.publishing.service.gov.uk/government/uploa ds/system/uploads/attachment data/file/453711/PHECRCE-017 maps with place names .pdf. Acesso em 25 mai2018.

Elío, J. (04 coautores), 2018. Estimation of residential radon exposure and definition of Radon Priority Areas based on expected lung cancer incidence. Env. Itnl, v. 114: 69-76, https://doi.org/10.1016/j.envint.2018.02.025.

Environment Protection Agency, EPA-I.E., 2018. Radon Risk. Disponível em http://www.epa.ie/radiation/radonmap/. Acesso em 25 mai2018.

Environment Protection Agency, EPA-U.S., 2018. EPA Map of radon zones. Acesso 25jun2018, disponível em: https://www.epa.gov/sites/production/files/201408/documents/colorado.pdf

_ 2015 The National Radon Action Plan - a strategy
saving lives. Disponível em: https://www.epa.gov/radon/national-radon-action-planstrategy-saving-lives. Acesso em 25mai2018.

EURATOM, 2013. Council directive on basic safety standards for protection against the dangers arising from exposure to ionising radiation. Disponível em https://ec.europa.eu/energy/en/topics/nuclearenergy/radiation-protection. Acesso em 25mai2018.

Godoy L., et al., (03 coautores), 2011. Distribuição de K, eU e eTh e avaliação da dose anual de radiação na região do granito Serra do Carambeí. 2011. Ver. Bras. Geof.., São Paulo 29(2): 359-375.

Gouvea et al. (03 coautores), 2014. Atividade regulatória do Radônio em minas subterrâneas. Simp. Latino- 
Americano sobre Radônio/ II Seminário sobre Radônio no Brasil. Soc. Bras. Prot. Radiol. Poços de Caldas.

Grastly R.L., Carson J.M., Charbonneau B.W., Holman P.B., 1984. Natural Background radiation in Canada. Geological Survey of Canada. Bulletin 360. 39p.

Guerra, M. R. et al (09 coautores), 2017. Magnitude e variação da carga da mortalidade por câncer no Brasil e Unidades da Federação, 1990 e 2015. Rev. Bras. Epidemiol., São Paulo, v. 20, supl. 1,: 102-115.

IAEA, 2010. Radioelement mapping: Nuclear Energy Series, No. NF-T-1.3. International Atomic Energy (IAEA). Vienna. $108 \mathrm{p}$.

2018. Design and conduct of indoor radon surveys -Draft Safety Report. Revised draft, 2018-02-19.DD. Reviewed by Tony Colgan and Olga German.

ICRP, 2009. Statement on Radon. Intnl Comm. on Radiological Protection. Disponível em www.icrp.org.

INCA, 2013. Diretrizes para a vigilância do câncer relacionado ao trabalho / Instituto Nacional de Câncer Jose Alencar Gomes da Silva; Org. Ribeiro, F. S. N., Otero, U. B.. - 2. ed. rev. e atual.- Rio de Janeiro: 192 p.

2017. $A B C$ do câncer: abordagens básicas para o controle do câncer / Instituto Nacional de Câncer José Alencar Gomes da Silva. Org. Silva, M.J.S. 3. ed. rev. atual. Rio de Janeiro: $108 \mathrm{p}$.

2018. Incidência de câncer no Brasil / Instituto Nacional de Câncer José Alencar Gomes da Silva. Coordenação de Prevenção e Vigilância. Rio de Janeiro: $126 \mathrm{p}$.

Jensen, C.L. et al. (04 autores), 2004. The Norwegian Radon Mapping and Remediation Program. In: Intnl. Radiat. Protect. Assoc. Congress, 11. Madrid, 2004

Merola, Y. L., 2017. Radiação natural e células tronco de tumores mamários em população residente no município de Poços de Caldas-MG. Tese de Doutorado, Universidade Estadual de Campinas. Campinas.

NGU-Geological Survey of Norway, 2018. Radon hazards. Página eletrônica https://www.ngu.no/en/topic/radon-hazards. Acesso em 25 mai2018.

Pascholati E.M., Amaral G., Osako L.D., 1997. Environmental radiation patter of the Lagoa Real uranium district, Bahia, Brazil". V Congresso internacional da sociedade brasileira de geofisica, Brazil, 1, 406-407.

Quarto et al. (04 coautores), 2015. Radon exposure assessment and relative effective dose estimation to inhabitants of Puglia region, South Italy. Int. J. Environ.
Res. Public Health, 12 (2015), pp. 14948-14957, 10.3390/ijerph121114948

Ray, K., Stick, M., 2015. Handbook of toxicology of chemical warfare agents, Chapter 2: radiation and health effects 2 Ed., Academic Press, p. 431-446.

Radiological Protection Institute of Ireland-RPII, 2002. Radon in Dwellings: the Irish national radon survey. Disponível em http://www.epa.ie/pubs/reports/radiation/RPII Radon Dw ellings Rad Surv 02.pdf. Acesso em 25mai2018.

Ruano-Ravina A, Fernández-Villar A, Barros-Dios JM. Radón residencial y riesgo de cáncer de pulmón en nunca fumadores. Arch. Bronconeumol. 2017, 53:475476.

UNSCEAR, 2000. United Nations Scientific Committee on the effects of atomic radiation. Vol.1: Sources, $82 \mathrm{p}$.

U.S. GEOLOGICAL SURVEY-USGS, 1993. Geologic radon potential of EPA region 8: Colorado; Montana; North Dakota; South Dakota; Utah; Wyoming. Open-File Report 93-292-H. Prepared in cooperation with the U.S. Environmental Protection Agency. 184 p. Disponível em https://pubs.usgs.gov/of/1993/0292h/report.pdf. Acesso em 25maio2018.

Veiga, L. H. S., 2004. Padrão de mortalidade em coorte histórica de trabalhadores expostos ao radônio em uma mineração subterrânea de carvão, Paraná, Brasil. Tese de Doutorado, Escola Nacional de Saúde Pública. Rio de Janeiro: s.n.

Watson et al. (04 coautores), 2014. A national radon hazard map of Norway based on geology and indoor radon. 12 Internat. Workshop on the Geol. Aspectos of Radon Risk Mapping, Prague.

WHO, 2009. Handbook on indoor radon: a public health perspective / Ed. Zeeb, H.; Shannoun, F. World Health Organization, Geneve, $94 \mathrm{p}$. 